\title{
Depuración de aguas residuales domésticas con Microorganismos eficientes en condiciones Altiplánicas en sistema mixto (anaerobio-aeróbio)
}

\author{
Purification of domestic wastewater with efficient microorganisms in highland condi- \\ tions in mixed system (anaerobic-aerobic) \\ Nancy Tania Mamani Ccama ${ }^{1}$ - Richard Davis Chavez Molina² - Juan Eduardo \\ Vigo Rivera ${ }^{3}$ - Rose Adeline Callata Chura ${ }^{4}$
}

\section{Resumen}

La investigación tiene por objetivo evaluar la depuración de aguas residuales domésticas en condiciones altiplánicas empleando Microorganismos Eficientes (ME) en un sistema mixto (anaerobio-aerobio). El sistema fue de un diseño mixto (anaerobio-aerobio) de flujo continuo, compuesto de un tanque séptico, sistema de aireación por bandejas y canal de oxidación; con tiempo de retención hidráulico de 15 días y con un caudal de $5.6 \mathrm{m3} /$ día, bajo condiciones ambientales no controladas. Los parámetros monitoreados fueron DBO5, SST y CTT cada 5 días. Se aplicó tres tratamientos: T1 (control), T2 (3L de ME), T3 (4L de $\mathrm{ME}$ ), mostrando que el tratamiento tres presenta mejores resultados con porcentajes de remoción de 78, 73 y 99.97\% de DBO5, SST y CTT, respectivamente. Se concluye que los Microorganismos Eficientes contribuyen en la disminución de la DBO5, SST y CTT en condiciones altiplánicas a 3850 msnm.

Palabras clave: Aguas residuales domésticas, microorganismos eficientes, remoción de materia orgánica y sistema aerobio.

\section{Abstract}

The objective of the research is to evaluate the treating domestic wastewater in highland conditions using Efficient Microorganisms (EM) in a mixed system (anaerobic-aerobic). The system was of a continuous flow mixed design (anaerobic-aerobic), composed of a septic tank, aeration system by trays and an oxidation channel; with hydraulic retention time of 15 days and with a flow of $5.6 \mathrm{m3} /$ day, under environmental conditions no controlled. It was monitored parameters of BOD5, SST and CTT every 5 days. They apply three treatments: T1 (control), T2 (3L of ME), T3 (4L of ME), showing that treatment three presents better results with removal percentages of 78,73 and $99.97 \%$ of BOD5, SST and CTT, respectively. It is concluded that Efficient Microorganisms decrease of BOD5, SST and CTT in highland conditions at 3850 masl.

Keywords: Altiplanic conditions, depuration, efficient microorganisms, wastewater.

\footnotetext{
${ }_{1}^{1}$ Ingeniero Ambiental. I.P.C.T Contratistas Generales S.R.L. nancymamani@upeu.edu.pe

2 Ingeniero Ambiental. I.P.C.T Contratistas Generales S.R.L. richard.davis.cm@gmail.com

${ }^{3}$ Maestro en Ingeniería Ambiental. Universidad Peruana Unión. eduardo.vigo@upeu.edu.pe

${ }^{4}$ Maestro en Tratamiento de Aguas y Reuso de desechos. Universidad Peruana Unión. rose.callata@upeu.edu.pe
} 


\section{Introducción}

Las aguas residuales (doméstica, municipales, industrial, agroindustriales, minero metalúrgicos y pluviales) son aquellas que han sufrido alteraciones físicas, químicas y biológicas (Hernández Muñoz, 2007) que contribuyen en la actualidad a la contaminación de recursos hídricos de los ecosistemas, afectando a la salud de la población por el inadecuado tratamiento y/o por descarga directa. Las aguas residuales domésticas, se caracterizan por contener alta materia orgánica (Carbonácea, proteínas y lípidos) (Rojas, 2002).

A nivel mundial y de Latinoamérica solo el $80 \%$ de las aguas residuales no tiene un tratamiento previo a su descarga, así en el Perú solo el 32\% de las aguas residuales que pasan por los alcantarillados reciben un tratamiento adecuado (OEFA, 2014).

Existen diferentes tecnologías de tratamiento de agua residual doméstica, para la remoción de materia orgánica, siendo tratamientos avanzados y convencionales (biológicos: aerobio - anóxico - anaerobio). Los procesos biológicos muestran eficiencia en la remoción de materia orgánica carbonosa medida (DBO5, DQO, COT, COT) asimismo por ser de bajo costo (Nodal, en Borges et al., 2012) la tecnología se considera como potencial de depuración en la viabilidad de tratamiento de aguas residuales (Grazia Luna, 2010).

Los microorganismos eficientes, denominados en inglés Effective Microorganism (EM), tecnología (Higa \& Parr, 1994), conformada por tres (3) grupos de bacterias: Fotosintéticas o fototróficas (Rhodopseudomonas sp), ácido lácticos (Lactobacillus sp) y levaduras (Saccharomyces $s p$ ) (Higa, 2001). Estos microorganismo tiene la alta capacidad de remoción de materia orgánica, eliminación de los malos olores (Pontaza Pivaral, 2014) Guatemala, está siendo operada por la Autoridad para el Manejo Sustentable del Lago de Amatitlán (AMSA y la facultad de trabajar bajo condiciones aerobias y anaerobias (Enríquez Brito, Viera Briones, \& Mendoza Garcia, 2010).

Existen diversas investigaciones de la tecnología de aplicación de los EM (Enríquez Brito et al., (2010), Agreda Montalico (2015), García Castro \& Robles García (2018) y Canales López \& Sevilla Carpio (2016)) que han mostrado la eficiencia en la depuración de aguas residuales domésticas. Por tanto, el objetivo de la investigación es evaluar la remoción de materia orgánica mediante un sistema combinado de anaerobio-aerobio con la utilización de microrganismos eficientes (EM) dentro del proceso biológico.

\section{Metodología}

\subsection{Lugar de estudio}

El proyecto se desarrolló durante tres (3) meses bajo condiciones no controladas en la Villa Chullunquiani, carretera Arequipa $\mathrm{Km} \mathrm{6}$, con las coordenadas geográficas UTM Este: 373169 y Norte: 8284153 a una altura de 3841 m.s.n.m.

\subsection{Tipo de estudio}

Las características de la investigación responden a un diseño de bloques completamente aleatorio (DBCA), con la aplicación del método de Dunnett y Duncan, para comparación de cada tratamiento con el testigo, con el objetivo de seleccionar un mejor tratamiento.

\subsection{Características del sistema de tratamiento de agua residual}

El sistema de tratamiento de agua residual doméstica se constituyó de: Tanque séptico, equipo de bombeo, bandejas de aireación, calefactor solar con EM y canal de maduración (ver Figura 1). 
Tabla 1.

Equipos para el sistema de tratamiento

\begin{tabular}{|c|c|}
\hline Equipos & Descripción \\
\hline Tanque séptico & $\begin{array}{l}\text { - Se utilizó la metodología del Reglamento Técnico del } \\
\text { Sector de Agua Potable y Saneamiento Básico de Co- } \\
\text { lombia RAS } 2000 \text { sección II capitulo E. } \\
\text { - La población contribuyente al sistema fue } 4 \text { habitantes. }\end{array}$ \\
\hline Equipo de bombeo & $\begin{array}{l}\text { - Se utilizó una bomba de marca SEBO con caudal de } \\
1200 \mathrm{~L} / \mathrm{H} \text { y una presión } 1.5 \mathrm{~m} \text {. }\end{array}$ \\
\hline Bandeja de aireación & $\begin{array}{l}\text { - Constituido por dos bandejas, cada bandeja tiene for- } \\
\text { ma trapezoidal con } 121 \text { orificios de diámetro } 1 / 8^{\prime \prime} \text {, con } \\
\text { diferencia de altura de } 30 \mathrm{~cm} \text {. }\end{array}$ \\
\hline Calefactor solar con EM & $\begin{array}{l}\text { - Se utilizó un diseño adaptado de SENCICO tipo muro } \\
\text { trombre. } \\
\text { - } \quad \text { El material utilizado para el diseño fue madera } \\
\text { El calefactor tiene la forma de un cubo. Solo un lado } \\
\text { está conformado por una superficie vidria. }\end{array}$ \\
\hline Canal de maduración & $\begin{array}{l}\text { - Se utilizó el diseño adaptado de la Corporación autóno- } \\
\text { ma regional de Nariño (2006). } \\
\text { El canal tiene } 7 \text { metros de longitud y presenta una } \\
\text { forma trapezoidal y tiene un cubierta de plástico en la } \\
\text { base para evitar la infirltración. }\end{array}$ \\
\hline
\end{tabular}

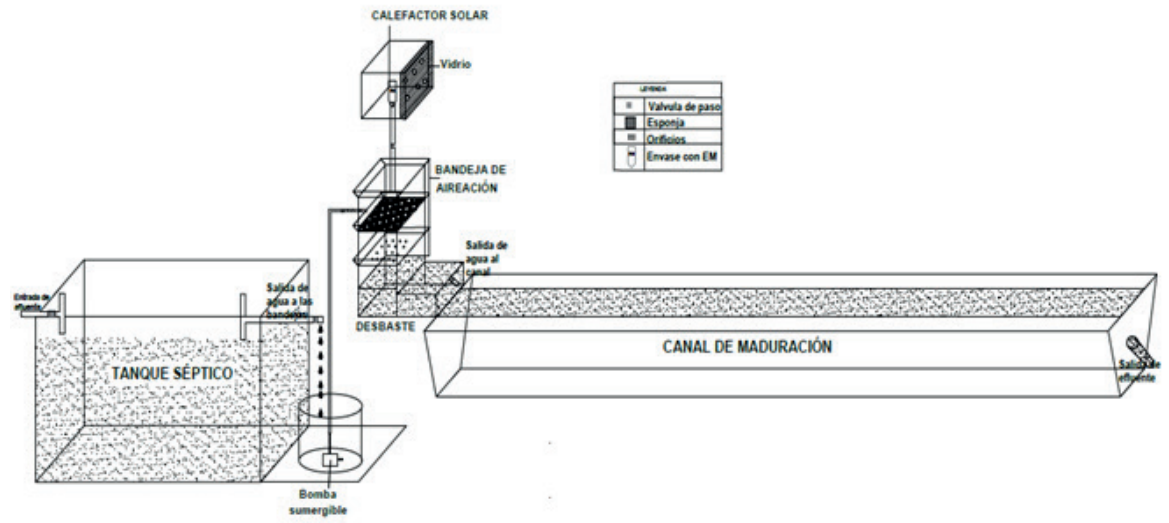

Figura 1. Diseño del sistema de tratamiento

\subsection{Tratamiento de aguas residuales}

El sistema de tratamiento de agua residual doméstica trabajó con un caudal de 5.60 m3/día, en el que se aplicaron microorganismos eficientes de 3 y $4 \mathrm{~L}$ de EM por cada ensayo, asimismo se consideró más un ensayo testigo (no se inoculo), con un tiempo de retención hidráulica de 15 días en condiciones ambientales no controladas, con análisis de DBO5, SST y CTT cada 5 días.

\subsection{Capacidad de remoción}

En la evaluación de la remoción se aplicó la siguiente ec. (Chuchón Martínez \& Aybar Escobar, 2008)se aplicó la técnica de Tubos Múltiples de Fermentación (NMP, en el análisis 
de los parámetros de DBO5, SST y CTT (Saboya Ríos, 2018) con la aplicación de microorganismos eficientes.

$$
\% \text { Eficiencia }=\frac{(\text { Concentración inicial-Concetración final })}{(\text { Concentración inicial })} * 100
$$

Antes del tratamiento del agua residual doméstica se caracterizó, donde se obtuvo concentraciones de DBO5, SST y CTT de 177 mg/L, 208 mg/L y 6.77x106 NMP/100 mL, respectivamente, datos que muestran un agua residual doméstica típica. La figura 2 muestra los resultados de la remoción de la de DBO5 de los tratamientos (0, 3 y 4 L de EM).

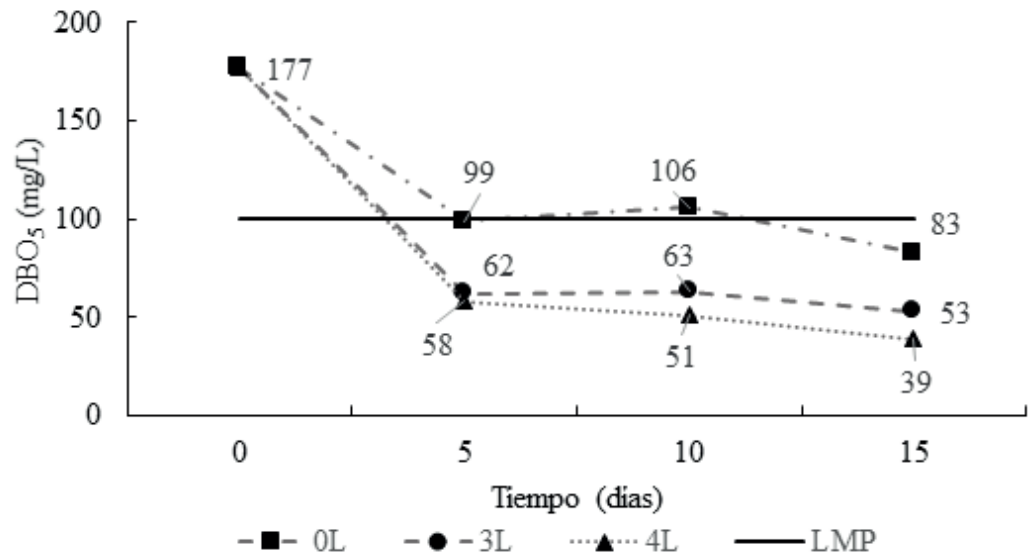

Figura 2. Comportamiento de la DBO5 con diferentes tratamientos y los LMP

El tratamiento con $4 \mathrm{~L}$ de EM muestra la mayor remoción de DBO5 con el 78\% (39 mg/L), valores que se encuentran por debajo de los Límites Máximo Permisible como efluente, asimismo, la prueba de Dunnet y Dunan indica que los tratamientos con EM son significativamente diferentes en su remoción, y superiores que el tratamiento testigo $(p<0.05)$. Lo que significa que la eficiencia del sistema aerobio ha sido influenciada por la presencia de los microorganismos eficientes en la degradación de la materia orgánica, según Ortiz (en Vargas Navas \& Turca Ceballos, 2006) a medida que el agua sea aireada durante 6 a 10 horas en presencia de un cultivo biológico (EM), la cantidad de microorganismos aumentan y de forma directa disminuye las cargas de DBO5. La bandeja de aireación potencializado con los microorganismos eficientes mejoran la remoción en un rango de 35\% al 64\% (Calderón Muñoz et al. 2012). La evaluación periódica de 5, 10 y 15 días permite observar que en un sistema aerobio más la presencia de los microorganismos eficientes pueden ser mejorados la calidad de un agua residual domésticas (Agreda Montalico, 2015) en diferentes condiciones ambientales.

Los resultados de los sólidos suspendidos totales se muestra en la figura 3, en un tiempo de evaluación de 15 días, donde se obtuvo una remoción variable por el sistema anaerobio-aerobio. 


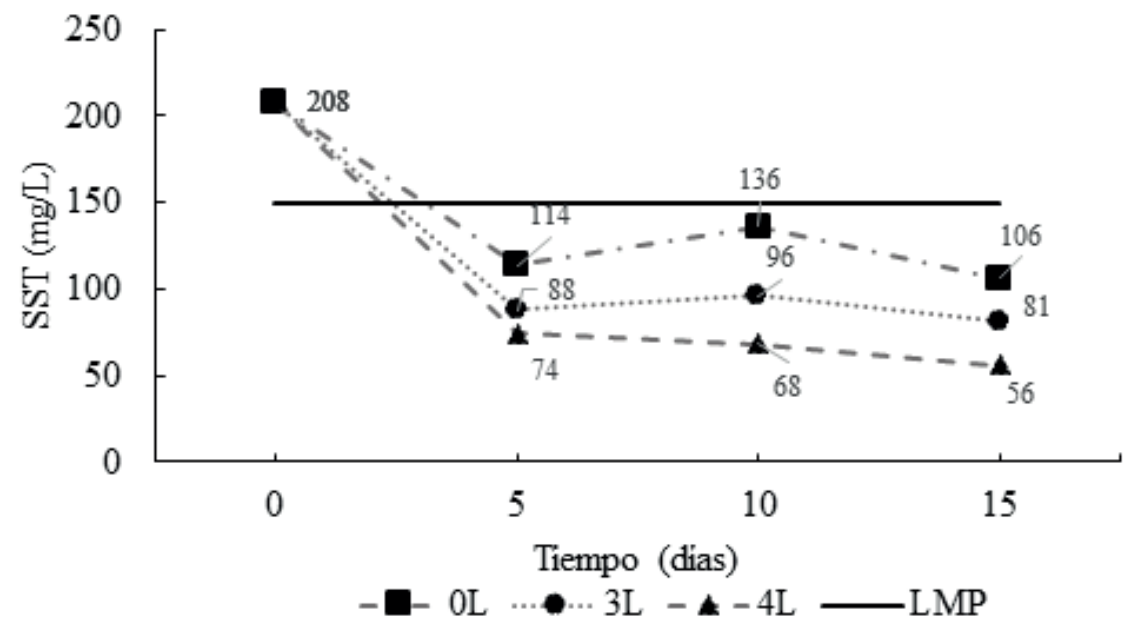

Figura 3. Comportamiento de SST con diferentes tratamientos y LMP

Los sólidos suspendidos totales señalan el comportamiento más significativo de la presencia de partículas suspendidas y solubilizadas, así en el tratamiento de aplicación de microorganismos eficientes de $4 \mathrm{~L}$ de EM se obtuvo una remoción del $73 \%$ (56 mg/L) resultado que muestra en comparación el tratamiento testigo de una remoción de $49 \%$ (106 mg/L). Asimismo, la prueba Dunnett indica que los tratamientos son significativamente diferentes y superiores que el tratamiento testigo $(p<0.05)$ y la prueba de Duncan reporta que de los tres grupos son significativamente diferentes, confirmando que la aplicación del EM es efectivo en la remoción de SST (Agreda Montalico, 2015), así pueden variar la eficiencia en función al volumen aplicado en la tratabilidad del agua residual.

Los resultados de los Coliformes Termotolerantes se muestran en la figura 4, en un tiempo de retención hidráulica de 15 días, en el que los microorganismos eficientes han actuado.

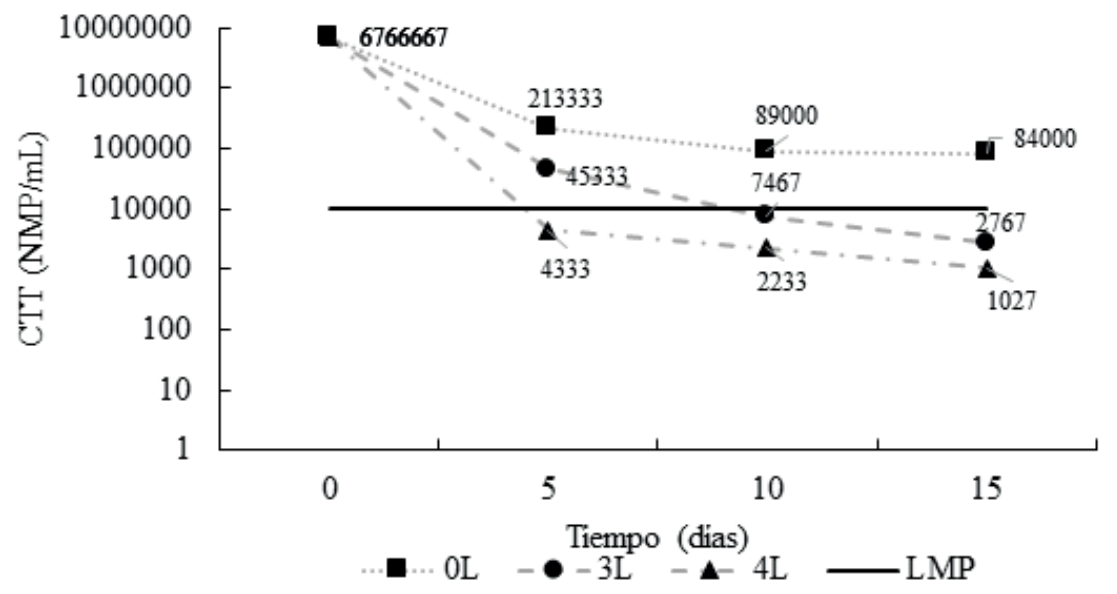

Figura 4. Comportamiento de CTT con diferentes tratamientos y LMP 
La remoción de los Coliformes Termotolerantes es influenciado por diferentes factores ambientales (radiaciones UV) y condiciones de tratabilidad del agua residual (profundidad del canal de maduración). También se tiene que el tiempo de retención hidráulica mejora la remoción de CTT, Von Munch (en Nuñez Burga, 2016) recomienda un tiempo de retención máximo de 6 a 7 días para una remoción del 98\% a 100\% de CTT

La aplicación de un volumen de $4 \mathrm{~L}$ de EM ha permitido mejorar el proceso de tratabilidad del agua residual doméstica con influencia en la remoción de los CTT en un 99.97\%, seguido de $99.96 \%$ ( $3 \mathrm{~L}$ de EM) y $98.76 \%$ (testigo). La prueba Dunnet y Duncan indica que los tratamientos son significativamente diferentes y superiores que el tratamiento testigo $(p<0.05)$. La aplicación de los microorganismos eficientes muestra que los EM eliminan y/o excluyen a los organismos nocivos y generan organismos beneficios que fortalecen al medio ambiente como las enzimas, ácidos orgánicos aminoácidos, hermanos y antioxidantes (Higa,2001; García Castro \& Robles García , 2018 y Espinoza Zegarra, 2017).

La prueba de ANOVA realizada para el análisis de SST y CTT muestra que no existe diferencia significativa $(p=0.081>0.05)$ entre los periodos de evaluación $(5,10$, y 15), en otras palabras, no existe una influencia directa entre los periodo de evaluación y remoción de los parámetros de SST y CTT. Por otro lado, los resultados pueden variar debido a las condiciones ambientales en que se apliquen (García Castro \& Robles García, 2018 y Valdez Pino, 2016) y el tiempo de retención hidráulica para la mejorar la remoción, Von Munch (en Nuñez Burga, 2016).

El pH varió (7.48 a 7.92; 7.4 a 8.33 y 6.15 a 7.88 para el tratamiento testigo, tratamiento de $3 \mathrm{~L}$ de $E M$, tratamiento de $4 \mathrm{~L}$ de EM, respectivamente) en función al crecimiento de microalgas en el canal de maduración así se observó que en los tres tratamientos presentan diferentes variaciones de $\mathrm{pH}$ en función también al volumen aplicado de microorganismos eficientes en la remoción de la materia orgánica. Por lo que, se encuentra en un rango de pH promedio de mejor actividad microbiana que permite la biodegradación más apropiada en el tratamiento de agua residual doméstica (Gil Rodríguez 2003).

La temperatura de operación fue en un promedio de 13.3 a $15.8^{\circ} \mathrm{C}$, valores que se encuentran en el rango de 10 a $35^{\circ} \mathrm{C}$ para el funcionamiento del tratamiento del agua residual de acuerdo, con Romero (en Nuñez Burga, 2016).

\section{Conclusiones}

La remoción de materia orgánica en un sistema anaerobio - aerobio con microorganismo eficientes (EM) implica condiciones ambientales y condiciones de diseño del sistema para alcanzar la mejor remoción de materia orgánica así se obtuvo una mejor remoción en el tratamiento de aplicación de $4 \mathrm{~L}$ de EM, con remociones al 78\% para la DBO5, 73\% para los SST y $99.97 \%$ para los CTT obtenido un promedio aproximado de $82.99 \%$ de eficiencia, lo que permite alcanzar con el cumpliendo de la normativa peruana del LMP en el vertimiento del efluente a un cuerpo receptor. La prueba Dunnett y Duncan mostraron que los tratamientos con EM en un sistema aerobio son significativamente diferentes $(p<0.0 .05)$ al testigo (sin EM) y los análisis de varianza muestran que no existe influencia significativa entre el periodo de evaluación y la remoción de CTT y SST, sin embargo si existe influencia significativa para remoción de DBO5 .

\section{Recomendaciones}

- En caso de que se deseara lograr una depuración más completa de DBO5, SST y CTT se recomienda que se implemente más bandejas de aireación, y los orificios de las bandejas sean más pequeñas. 
- Realizar estudios en épocas de invierno, a fin de observar si el comportamiento del sistema de tratamiento es similar.

- Se recomienda determinar los parámetro físico, quimos y microbiológicos en un periodo mayor a los 15 días, ya que los resultados demostraron una mejor eficiencia al pasar de los tiempos.

\section{Bibliografía}

Agreda Montalico, K. I. (2015). Evaluación de la efectividad de microorganismos eficaces en las propiedades físico químicas del agua residual de la planta de tratamiento, a nivel laboratotio, Ilo 2014. Universidad Nacional de Moquegua.

APHA, AWWA, \& WPCF. (2005). Metodos normalizados para el analisi de agua potable y residuales,APHA-AWWA-WPCF. (A. Lenore s. Clesceri, wpcf, presidente arnold e. Greenberg, apha r. Rhodes trussell, Ed.) (Dias de Sa). Madrid-España.

Borges, C., Elba, R., Rojas, B., Novelo, M., Roger, I., Rodríguez, O., ... Novelo, R. I. M. (2012). Remoción de materia orgánica en aguas residuales de rastro por el proceso de Contactor Biológico Rotacional. Ingeniería, 16(2), 83-91.

Calderón Muñoz, R. F., Forero Poveda, S. del P., \& Suarez Cerquera, A. (2012). Implementación de un diseño piloto de bandejas de aireación para aguas, potencializado con microorganismos eficientes. Sección Ciencia e Ingeniería, 16, 23-35. https://doi.org/0124 2253

Canales López, H. O., \& Sevilla Carpio, A. A. (2016). Evaluación del uso de microorganismos eficaces en el tratamiento de efluentes domésticos residuales del distrito de PÁTAPO. Universidad Nacional "Pedro Ruiz Gallo."

Chuchón Martínez, S. A., \& Aybar Escobar, C. A. (2008). Evaluacion de la capacidad de remocion de bacterias coliformes fecales y DBO de la planta de tratamiento de aguas residuales "La Totora", Ayacucho. Ecología Aplicada, 7, 166-171. https://doi.org/17262216

Corporación autonoma regional de nariño. (2006). Sistema de tratamiento de aguas residuales industriales para trapiches paneleros.

Enríquez Brito, J. L., Viera Briones, J. L., \& Mendoza Garcia, F. (2010). Caracterización preliminar de aislamiento de Microorganismos, mediante la técnica de E . M ., a nivel de comunidades vegetales en dos zonas de vida ecológicamente diferentes. Retrieved from http://www.dspace.espol.edu.ec/bitstream/123456789/9017/1/Caracterización preliminar de aislamiento de microorganismos.pdf

Espinoza Zegarra, A. E. (2017). Disminución de la DBO, DQO y STD del agua residual domestica de Santiago de Chuco empleando un biofiltro de piedra pómez. Retrieved from http://www.ptolomeo.unam.mx:8080/xmlui/bitstream/handle/132.248.52.100/3547/ TESIS Viscosidad \%28Bibliotecas UNAM\%29.pdf?sequence=2

García Castro, Y. C., \& Robles García, D. E. (2018). Determinación de la dosis de microorganismos eficientes para el tratamiento de aguas residuales domésticas provenientes de la universidad nacional de Ucayali, distrito de Callería, provincia de coronel portillo, Ucayali.

Gil Rodríguez, M. (2003). Cálculos avanzados en procesos de descontaminación de aguas (Consejo Su). Madrid-España. 
Grazia Luna, R. M. (2010). Oportunidades de mejoras ambientales por el tratamiento de aguas residuales en el Peru. Grupos Relaciona, 1-37. Retrieved from www.fonamperu. org/.../agua/.../Oportunidades_Mejoras_Ambientales.pd...

Hernández Muñoz, A. (2007). Saneamiento y Alcantarillado Vertidos Residuales. (Seinor, Ed.). España.

Higa, T. (2001). The technology of effective microorganisms-concept and philosophy. University of the Ryukyus, Okinawa, Japan. Retrieved from http://naturefarmingmada.org/ img/article/pdf/EM_concept.pdf

Higa, T., \& Parr, J. F. (1994). Beneficial and Effective Microorganisms for a Sustainable Agriculture and Enviroment. Agriculture, (808), 1-16.

Nuñez Burga, R. M. (2016). Tratamiento de aguas residuales domésticas a nivel familiar, con Humedales Artificiales de flujo subsuperficial Horizontal, mediante la especie macrófita emergente Cyperus Papyrus (Papiro). Universidad Peruana Unión.

OEFA. (2014). OEFA prioriza fiscalización ambiental del manejo de aguas residuales. Perú-Lima.

Pontaza Pivaral, J. M. (2014). Eficiencia de microorganismo efectivos (EM) aplicados en la planta de tratamiento de aguas residuales San Cristobal (Colonia Panorama),Mexco,Guatemala. Universidad de San Carlos de Guatemala. Retrieved from http://sitios.ingenieria.usac.edu.gt/eris/tesis_eris/is/IS_0262.pdf

Rojas, R. (2002). Conferencia Sistemas de Tratamiento de Aguas Residuales.

Saboya Ríos, X. V. (2018). Eficiencia del método de lombrifiltro en la remoción de los contaminantes de las aguas residuales domésticas en el Distrito de Chachapoyas-Amazonas. Universida Peruna Unión. https://doi.org/10.1587/transcom.E93.B.2477

Valdez Pino, A. (2016). Aplicación de microorganismos eficaces (EM)para el tratamiento de las aguas residuales domesticas en la localidad de Chucuito. Universidad Nacional del Altiplano. Universidad Nacional del Altiplano.

Vargas Navas, O. H., \& Turca Ceballos, J. (2006). Comportamiento de la eficiencia de remoción de materia Orgánica en un bioreactor de lecho móvil alimentado con agua residual doméstica, con la variación del volumen del lecho. Universidad la salle. Universidad la salle.

WWAP. (2017). Informe Mundial de las Naciones Unidas sobre el Desarrollo de los Recursos Hídricos 2017. Aguas Residuale: el recurso desaprovechado. Paris. 